\title{
Modification of Rhizosphere Bacterial Community Structure and Functional Potentials to Control Pseudostellaria heterophylla Replant Disease
}

\author{
Linkun Wu, 1,2 Bo Yang, ${ }^{1,2}$ Manlin Li, ${ }^{1,2}$ Jun Chen, ${ }^{1,2}$ Zhigang Xiao, ${ }^{1,2}$ Hongmiao Wu, ${ }^{1,2}$ Qingyu Tong, ${ }^{1,3}$ \\ Xiaomian Luo, ${ }^{2,3}$ and Wenxiong Lin ${ }^{1,3, \dagger}$ \\ ${ }^{1}$ College of Life Sciences, Fujian Agriculture and Forestry University, Fuzhou 350002, Fujian, China \\ ${ }^{2}$ Key Laboratory of Crop Ecology and Molecular Physiology, Fujian Agriculture and Forestry University \\ ${ }^{3}$ Fujian Provincial Key Laboratory of Agroecological Processing and Safety Monitoring, Fujian Agriculture and Forestry \\ University
}

\begin{abstract}
Replant disease caused by negative plant-soil feedback commonly occurs in a Pseudostellaria heterophylla monoculture regime. Here, barcoded pyrosequencing of $16 \mathrm{~S}$ ribosomal DNA amplicons combined with phylogenetic investigation of communities by reconstruction of unobserved states (PICRUSt) analysis was applied to study the shifts in soil bacterial community structure and functional potentials in the rhizosphere of $P$. heterophylla under consecutive monoculture and different soil amendments (i.e., bio-organic fertilizer application $[\mathrm{MF}]$ and paddy-upland rotation $[\mathrm{PR}])$. The results showed that the yield of tuberous roots decreased under $P$. heterophylla consecutive monoculture and then increased after MF and PR treatments, which was consistent with the changes in soil bacterial diversity. Both principal coordinate analysis and the unweighted pair-group method with arithmetic means cluster analysis showed the distinct difference in bacterial community structure between the consecutively monocultured soil (relatively unhealthy soil) and other relatively healthy soils (i.e., newly planted soil, MF, and PR). Furthermore, taxonomic analysis showed that consecutive monoculture of $P$. heterophylla significantly decreased the relative abundances
\end{abstract}

Abstract of the families Burkholderiaceae and Acidobacteriaceae (subgroup 1), whereas it increased the population density of families Xanthomonadaceae, Phyllobacteriaceae, Sphingobacteriaceae, and Alcaligenaceae, and Fusarium oxysporum. In contrast, the MF and PR treatments recovered the soil microbiome and decreased $F$. oxysporum abundance through the different ways; for example, the introduction of beneficial microorganisms (in MF) or the switching between anaerobic and aerobic conditions (in PR). In addition, PICRUSt analysis revealed the higher abundances of membrane transport, cell motility, and DNA repair in the consecutively monocultured soil, which might contribute to the root colonization and survival for certain bacterial pathogens under monoculture. These findings highlight the close association between replant disease of $P$. heterophylla and the variations in structure and potential functions of rhizosphere bacterial community.

Keywords: cultural and biological practices, disease management, fungi, herbaceous/flowering plants, ornamentals, pathogen diversity, prokaryotes
Plant-soil feedbacks (PSF) refer to the changes to soil properties that are caused by plants which, in turn, influence the performance of plants (van der Putten et al. 2013). Up to $20 \%$ of plant photosynthates may be released into the rhizosphere by plant roots, which shape the root-associated microbiome (Lareen et al. 2016; Lundberg and Teixeira 2018; Poole 2017). In turn, the root-associated microbiome is a key modulator for soil health and plant performance (Berendsen et al. 2012; Hu et al. 2018). Plant responses to PSF can be negative, neutral, or positive (Kaisermann et al. 2017; van der

${ }^{\dagger}$ Corresponding author: W. Lin; lwx@ fafu.edu.cn

L. Wu, B. Yang, and M. Li contributed equally to this work.

Funding: This work was supported by grants from the Natural Science Foundation of Fujian Province (number 2017J01803), the Outstanding Youth Scientific Fund of Fujian Agriculture and Forestry University (number XJQ201501), the Science and Technology Innovation Fund of Fujian Agriculture and Forestry University (number CXZX2017302), the Program for Innovative Research Team in Fujian Agricultural and Forestry University (number 712018009), the National Natural Science Foundation of China (numbers 81573530 and 81303170), and Fujian-Taiwan Joint Innovative Center for Germplasm Resources and Cultivation of Crop (FJ 2011 Program, number 2015-75).

*The $\boldsymbol{e}$-Xtra logo stands for "electronic extra" and indicates that five supplementary figures and one supplementary table are published online.

The author(s) declare no conflict of interest.

Accepted for publication 16 August 2019.

(c) 2020 The American Phytopathological Society
Putten et al. 2013). Replant disease or soil sickness, a typical negative PSF, is featured with a decline in crop yield and a prevalence of soilborne diseases as a result of repeated planting of the same plant species in the same soil for multiple years (Huang et al. 2013). Replant disease caused by negative PSF commonly occurs in the monoculture cultivation of a range of crops in intensive agriculture such as horticultural crops and medicinal plants (Tian et al. 2016; L. K. $\mathrm{Wu}$ et al. 2016a; Zhang et al. 2011). In particular, approximately $70 \%$ of medicinal plant species with tuberous roots suffer from replant disease when grown in monoculture (Chen et al. 2015; L. K. $\mathrm{Wu}$ et al. 2016a). Pseudostellaria heterophylla, an important traditional Chinese medicine (TCM), is commonly used for curing inappetence, debility, diabetes, and weakness after illness (Hua et al. 2016). However, consecutive monoculture of $P$. heterophylla results in a severe decline in both quality and yield of tuberous roots, the most useful part in TCM (L. K. Wu et al. 2016b).

Soil microbial community structure and functions are of crucial importance in determining soil quality and ecosystem stability and sustainability (Schloter et al. 2003; Yang et al. 2017; Zhao et al. 2014). Various studies demonstrated the distinct difference in soil bacterial communities between disease-suppressive soils and disease-conducive soils (Carrión et al. 2018; Gómez Expósito et al. 2017; Kyselková et al. 2009; Li et al. 2015). Mendes et al. (2011) attributed soil suppressiveness of Rhizoctonia solani to the high abundances of several bacterial taxa such as Pseudomonadaceae, Burkholderiaceae, and Xanthomonadales in the sugar beet rhizosphere. Shen et al. (2015a) pointed out that soils naturally suppressive to banana Fusarium wilt disease harbored unique bacterial communities, with higher abundances of the phylum Acidobacteria and the genera Chthonomonas, Pseudomonas, and Tumebacillus. In recent years, increasing studies using different crops demonstrated that the occurrence of replant disease was closely related to the shifts 
in soil bacterial communities (Dong et al. 2016; Li et al. 2014a; Mazzola and Manici 2012; Tan et al. 2017; Xiong et al. 2015; Zhou and $\mathrm{Wu} 2018$ ). Likewise, our previous studies using denaturing gradient gel electrophoresis found that consecutive monoculture of $P$. heterophylla greatly altered the structure of rhizosphere bacterial community with a reduction in the abundance of beneficial rhizobacteria and resulted in the declined soil suppressiveness to Fusarium oxysporum, which showed a high pathogenicity to $P$. heterophylla (Chen et al. 2017; L. K. Wu et al. 2016b; Zhao et al. 2015). Therefore, restructuring the rhizosphere microbial community has become the key point for improving soil quality and alleviating $P$. heterophylla replant disease.

Currently, some approaches, including biological control by adding bio-organic fertilizer and crop rotation were widely applied in intensive agriculture for the protection of plants against soilborne pathogens (Hou et al. 2018; Li et al. 2018; Ling et al. 2014; Shen et al. 2015b; Wang et al. 2016, 2017). Reestablishment of soil suppressiveness to soilborne diseases by bio-organic fertilizer application was attributed not only to general suppression by increasing soil microbial diversity and activity but also to specific suppression by fostering specific beneficial microorganisms (Ling et al. 2014; Qiu et al. 2012). By contrast, crop rotation was reported to affect soil microbial community structure by increasing temporal plant diversity and increasing microbial diversity by providing a greater concentration and diversity of organic materials in comparison with the monoculture system (Peralta et al. 2018; Yao et al. 2006; Zhou et al. 2017). Zhao et al. (2017) indicated that, in comparison with Panax notoginseng monoculture, crop rotation could enhance the soil microenvironment for the growth of more abundant and diverse soil microbial communities, increase the bacterial diversity and the abundance of beneficial microorganisms (i.e., Bacillus spp.), and decrease the ratio of $F$. oxysporum/fungi in soil. Furthermore, Latz et al. (2012) demonstrated that the abundance of soil bacteria producing the antifungal compounds 2,4-diacetylphloroglucinol and pyrrolnitrin increased with plant diversity, suggesting that plant diversity contributes to plant community resistance against pathogens by fostering a beneficial bacterial community. In addition, crop rotation between flooded and upland soil conditions could alter soil microbial community structure and functions by regulating the soil physicochemical properties (i.e., soil aggregate, redox potential, and soil pH) (Breidenbach et al. 2017; Gaydon et al. 2012; Hou et al. 2018; Zhou et al. 2014). Breidenbach et al. (2017) demonstrated the different soil microbial communities in flooded rice versus upland maize soil, mainly due to the different soil environmental conditions (i.e., anoxic flooded soil supporting an anaerobic microbial community versus oxic drained soil supporting an aerobic microbial community).

In this study, both microbial fertilizer application and paddyupland rotation were carried out in Pseudostellaria heterophylla monoculture regimes to assess their efficacy on controlling replant disease. Moreover, deep pyrosequencing of $16 \mathrm{~S}$ ribosomal DNA (rDNA) amplicons combined with phylogenetic investigation of communities by reconstruction of unobserved states (PICRUSt) analysis was applied to study the bacterial community structure and functional responses to different soil amendments. We hypothesized that both microbial fertilizer application and paddy-upland rotation could recover the soil microbiome by promoting bacterial diversity and modulating specific microbial composition.

\section{Materials and Methods}

Field experiment and sampling. P. heterophylla 'Zheshen 2' was used as the test plant material in this study. P. heterophylla was generally planted in November and harvested in July of the next year. The field experiment was carried out at Guanyang County, Fuding City, Fujian Province, China $\left(27^{\circ} 25^{\prime} \mathrm{N}, 120^{\circ} 04^{\prime} \mathrm{E}\right)$. This region is a subtropical monsoon climate with the annual mean precipitation of 2,041 mm. Five treatments were carried out in a field previously cultivated with rice (Oryza sativa). The timeframe for experiments and sampling is presented in Figure 1. Experiments included a control with no $P$. heterophylla cultivation during the whole experimental period (CK), newly planted plots (NP), 2-year consecutively monocultured plots (CM), 1-year cultivated plots first treated with the microbial fertilizer FAFU and then replanted with $P$. heterophylla (MF), and $P$. heterophylla-rice-P. heterophylla rotation plots (PR). Among them, microbial fertilizer FAFU consisted of effective microorganisms (consisting of lactic acid bacteria, yeast, actinomycetes, photosynthetic bacteria, and certain fungi) (Xu 2001) and indigenous antagonistic bacteria previously isolated from $P$. heterophylla rhizosphere (Burkholderia, Pseudomonas, and Bacillus spp.). These indigenous antagonistic bacteria show strong antagonistic activities against the soilborne pathogens of $P$. heterophylla (i.e., $F$. oxysporum, F. moniliforme, and Talaromyces helices) (Chen et al. 2017; H. M. Wu et al. 2017; L. K. Wu et al. 2016a,b). The bio-organic fertilizer was prepared by incubating a mixture of these beneficial microorganisms in an organic mixture of soybean meal and fish meal $(2: 1, \mathrm{wt} / \mathrm{wt})$ according to the solid fermentation method (L. K. Wu et al. 2016a). The solid-state fermented fertilizer (480 kg per $666.7 \mathrm{~m}^{2}$ ) was applied in the 1-year $P$. heterophylla-cultivated plots on 14 October 2014 according to L. K. Wu et al. (2016a). All five treatments had three replicate plots and the study plots were closely adjacent to each other to keep the same environmental conditions. Moreover, all treatments were kept under the same field management during the whole experimental period.

At the expansion stage of tuberous roots (15 May 2015), the rhizosphere soils of $P$. heterophylla (NP, CM, MF, and PR) and control soils (CK) were sampled. For rhizosphere soil sampling, the whole plants were carefully dug out of the soil and shaken to remove loosely attached bulk soil. The soil tightly attached to tuberous roots, called

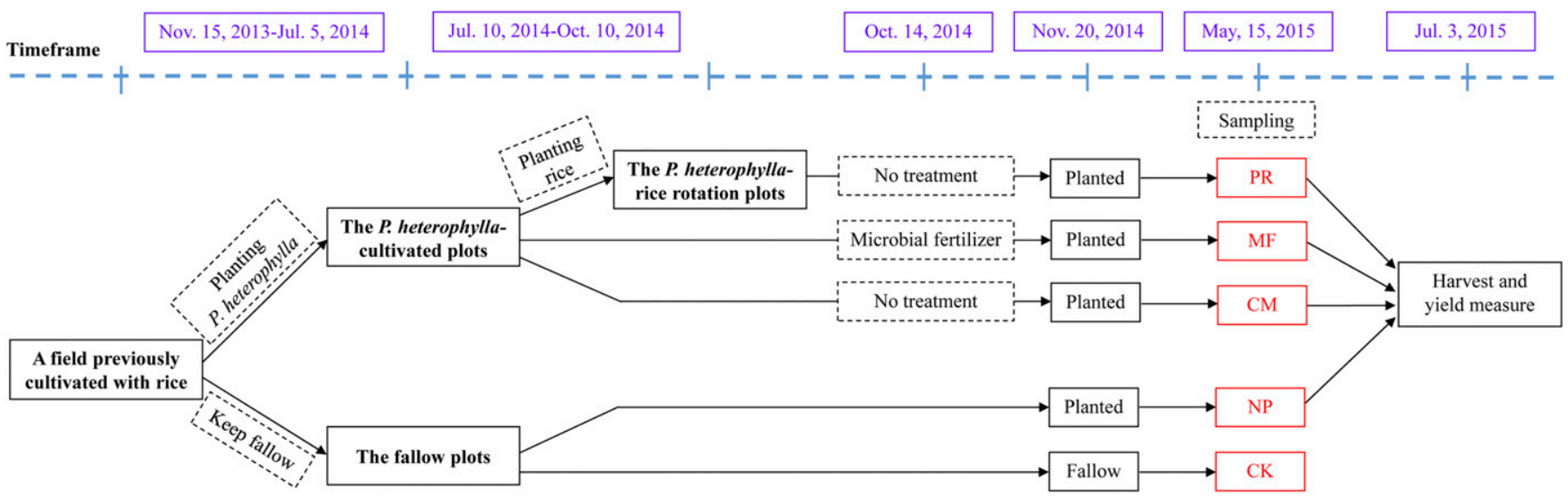

Fig. 1. Timeframe for the field experiment and soil sampling. CK, NP, and CM represent the control with no Pseudostellaria heterophylla cultivation, the newly planted plots, and 2year monocultured plots, respectively. MF represents the 1 -year cultivated plots first treated with the microbial fertilizer FAFU and then replanted with $P$. heterophylla. PR represents P. heterophylla-rice-P. heterophylla rotation plots. 
rhizosphere soil, was brushed off and then collected. Each treatment had three biological replicates from the replicate plots. All soil samples were sieved through 2-mm mesh. A portion of soil was stored at $-80^{\circ} \mathrm{C}$ for the extraction of soil total DNA. The rest of the soil for each treatment was air dried and used for soil nutrient determination according to the methods described by L. K. Wu et al. (2016a). On 3 July 2015, the tuberous roots of $P$. heterophylla from all treatments (NP, CM, MF, and PR) were separately dug out, washed, and sun dried, after which the dry weight was measured.

Soil total DNA extraction, PCR amplification, and barcoded pyrosequencing. The extraction of soil total DNA was performed by using a BioFast soil Genomic DNA Extraction kit (BioFlux, Hangzhou, China). The quality of extracted soil DNA was checked on $1 \%$ agarose gels and the DNA concentrations from different soil samples were determined at the same time by using a Nanodrop 2000C Spectrophotometer (Thermo Scientific, Waltham, MA, U.S.A.). Then, high-quality soil DNA was used for the amplification of variable regions 3 to 4 of bacterial $16 \mathrm{~S}$ rDNA gene via the primers $341 \mathrm{~F}\left(5^{\prime}-\right.$ CCTAYGGGRBGCASCAG-3') and 806R (5'-GGACTACNNGGG TATCTAAT- $3^{\prime}$ ) with the unique barcode for each sample.

The PCR product from each sample was equally mixed and then used to construct the sequencing library via a TruSeqDNA PCRFree Sample Preparation Kit (Illumina, San Diego, CA, U.S.A.). The high-quality library was sequenced using a HiSeq2500 platform (Illumina, San Diego, CA, U.S.A.) and 250-bp paired-end reads were obtained.

Processing of pyrosequencing data. The paired-end reads within each sample were separated by the unique barcode and then truncated by removing the barcode and primer sequences. Sequence assembly of the paired-end reads was performed using FLASH software (v1.2.7) (Magoč and Salzberg 2011). The low-quality reads were filtered out using Trimmomatic software (v0.33) (Bolger et al. 2014) and the chimeric sequences were removed using UCHIME software (v4.2) (Edgar et al. 2011) to obtain the effective reads (SRA accession PRJNA554972). The effective reads were binned into operational taxonomic units (OTUs) using QIIME (version 1.8.0) (Caporaso et al. 2010), with 97\% similarity. Species annotation were performed by using RDP classifier (version 2. 2; https://sourceforge. net/projects/rdp-classifier/) (Wang et al. 2007) against the SILVA database (release128; https://www.arb-silva.de) (Quast et al. 2012).

Furthermore, $\alpha$ diversity (OTU number, Pielou's evenness, and Shannon diversity indices) was calculated to analyze species complexity within a sample. The $\beta$ diversity analysis, including principal coordinate analysis (PCoA) and the unweighted pair-group method with arithmetic means (UPGMA) clustering based on the BrayCurtis distance, was applied to analyze species complexity differences between samples. In addition, the PICRUSt approach (Langille et al. 2013) was applied in this study to predict the functional composition of a metagenome based on a $16 \mathrm{~S}$ profile.

Quantitative PCR of $\boldsymbol{F}$. oxysporum in different soil samples. The abundance of $F$. oxysporum, serving as an indicator of $P$. heterophylla replant disease in a monoculture regime (Chen et al. 2017; L. K. Wu et al. 2016b; Zhao et al. 2015), in different soil samples, was determined through quantitative PCR (qPCR) with the speciesspecific primers ITS1-F (5'-CTTGGTCATTTAGAGGAAGTAA-3') and AFP308R (5'-CGAATTAACGCGAGTCCCAA-3') (Lievens et al. 2005). The PCR mixture and amplification conditions were conducted according to L. K. Wu et al. (2015).

Statistical analyses. One-way analysis of variance (ANOVA) followed by a least significant difference test $(P<0.05)$ was carried out to determine the significance of yield of tuberous roots, abundance of specific taxa, soil physicochemical parameters, and $\alpha$ diversity indices among different treatments. Extended errors bars were performed in The Statistical Analysis of Metagenomic Profiles software (Parks et al. 2014) with Welch's $t$ test and Welch's inverted confidence interval method to determine significant differences between two groups for taxonomic (at family level) and functional (at KEGG level 2) profiles. $P$ values for taxonomic profiles were adjusted with Benjamini-Hochberg false discovery rate (FDR) correction (Pacchioni et al. 2014) and denoted $q$ values $(q<0.05)$. Statistical differences for KEGG pathways (level 3) were estimated by ANOVA with Tukey-Kramer post hoc test using a Benjamini-Hochberg FDR correction (the parameters for filtering out were as follows: $q$ value $>$ 0.05 , effect size $<0.8$ ). Similarity percentage (SIMPER) analysis based on the relative abundances of genera was performed to determine the top taxa contributing to the dissimilarities between samples by using the PRIMER V5 software package (PRIMER-E Ltd., Plymouth, U.K.).

\section{Results}

The growth status of $P$. heterophylla under different treatments. Compared with the NP treatment, the CM treatment seriously affected $P$. heterophylla above- and belowground growth with a significant $(P<0.05)$ decline in the yield of tuberous roots (Fig. 2A and B). The MF and PR treatments significantly $(P<0.05)$ improved the yield of tuberous roots as compared with $\mathrm{CM}$. There was no significant difference in yield between NP and MF but the yield in NP was significantly $(P<0.05)$ higher than that in PR (Fig. 2B). In addition, the abundance of $F$. oxysporum, an indicator of $P$. heterophylla replant disease, significantly $(P<0.05)$ increased in $\mathrm{CM}$ and then significantly $(P<0.05)$ decreased in PR and MF (Fig. 2C). Pearson correlation analysis revealed that the yield of tuberous roots was significantly negatively correlated with the abundance of $F$. oxysporum $(r=-0.918, P<0.01)$.

The soil physicochemical parameters under different treatments. Compared with the NP treatment, the CM treatment showed a significant $(P<0.05)$ increase in total and available $\mathrm{N}$, total $\mathrm{P}$, and available $\mathrm{K}$. Compared with the $\mathrm{CM}$ treatment, the MF treatment showed a significant $(P<0.05)$ decrease in total and available $\mathrm{N}$ and total and available $\mathrm{P}$ but a significant $(P<0.05)$ increase in total and available K. The highest available $\mathrm{N}$, total and available $\mathrm{P}$, total and available $\mathrm{K}$, and $\mathrm{pH}$ were found in the PR treatment. As for soil $\mathrm{pH}$, the $\mathrm{CM}$ treatment significantly $(P<0.05)$ decreased the $\mathrm{pH}$ value in the $P$. heterophylla rhizosphere, whereas the MF and PR treatments significantly $(P<0.05)$ increased the $\mathrm{pH}$ value (Table 1).

Overview of 16S rDNA genes pyrosequencing. In total, 803,341 effective tags were generated from 15 soil samples; 57,597, 55,225,
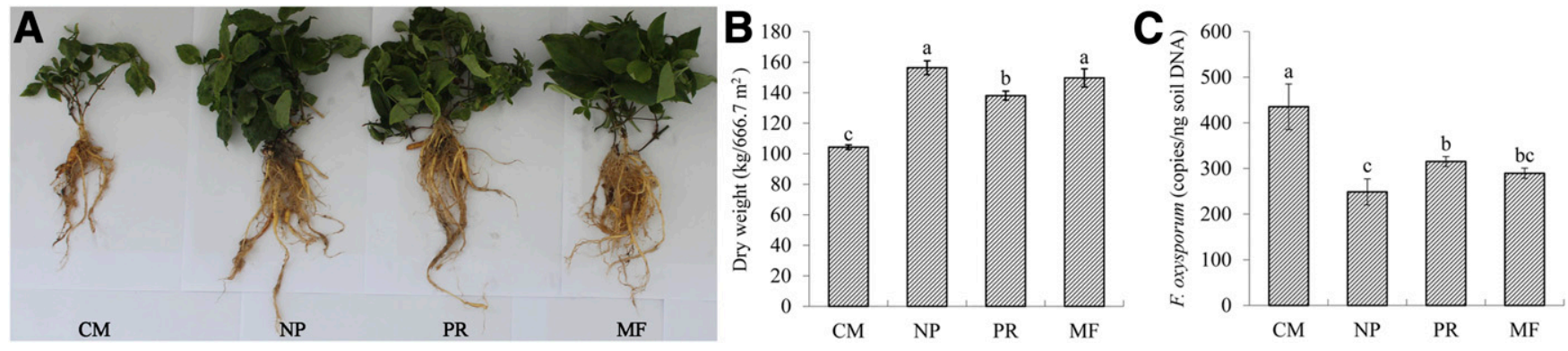

Fig. 2. A, Growth status; B, tuberous root yield; and C, rhizosphere Fusarium oxysporum abundance of Pseudostellaria heterophylla under different treatments. NP and CM represent the newly planted and 2-year monocultured plots, respectively, PR represents $P$. heterophylla-rice-P. heterophylla rotation plots, and MF represents the 1-year cultivated plots first treated with the microbial fertilizer FAFU and then replanted with $P$. heterophylla. 
$51,893,51,123$, and 51,943 effective tags were obtained from CK, $\mathrm{NP}, \mathrm{CM}, \mathrm{MF}$, and PR, respectively. Rarefaction analysis showed that the observed species number tended to reach a saturation level at 40,000 sequences (Supplementary Fig. S1). An OTU cluster showed that $1,590,1,603,1,581,1,582$, and 1,587 OTUs were obtained from CK, NP, CM, MF, and PR, respectively. On average, 89.3, 89.2, 88.5, $87.6,83.8$, and $68.7 \%$ of effective tags could be assigned to the phylum, class, order, family, genus, and species levels, respectively.

Bacterial $\boldsymbol{\alpha}$-diversity analysis. CK showed the highest OTU number, evenness, and diversity indices (Pielou's evenness and Shannon diversity indices), while CM showed the lowest OTU number, evenness, and Shannon diversity indices (Fig. 3). All three indices decreased in $\mathrm{CM}$ and then increased after MF and PR treatments (Fig. 3). PR showed a significantly $(P<0.05)$ higher OTU number, evenness, and diversity indices in comparison with CM (Fig. 3).

PCoA and cluster analysis. Both PCoA and UPGMA cluster analysis showed a distinct difference in soil bacterial community structure between the unhealthy soils (i.e., CM) and the relatively healthy soils (i.e., CK, NP, MF, and PR) (Fig. 4). The first two principal components (PC1 and PC2) of PCoA explained 57.43 and $11.78 \%$ of the total variations, respectively. $\mathrm{CM}$ was separated from the other soil samples across the first axis (Fig. 4A). Cluster analysis showed that $\mathrm{CK}$ and PR, and NP and MF, were clustered together, respectively, which were considerably different from the CM treatment (Fig. 4B).

Shifts in rhizosphere bacterial community structure under different treatments. Taxonomy analysis showed that phyla Proteobacteria, Acidobacteria, Thaumarchaeota, and Actinobacteria were dominant in all samples. The relative abundances of phyla Proteobacteria and Bacteroidetes were significantly $(P<0.05)$ higher in $\mathrm{CM}$ than in other treatments. However, the relative abundances of phyla Acidobacteria, Chloroflexi, Nitrospirae, and Verrucomicrobia decreased in CM and then increased after MF and PR treatments (Supplementary Fig. S2).
Furthermore, Venn diagram analysis based on the OTUs that were more abundant in all healthy soils (NP, MF, and PR) than in unhealthy soils $(\mathrm{CM})$ showed that 826 OTUs were shared among NP > $\mathrm{CM}$, MF > CM, and PR > CM (Supplementary Fig. S3). The sum of the relative abundances of these 826 OTUs was higher than $60 \%$ in all samples except CM (25.93\%). These 826 OTUs that were more abundant in healthy soils (NP, MF, and PR) were mainly assigned to the phyla Proteobacteria, Acidobacteria, Thaumarchaeota, and Actinobacteria. In addition, the shifts in the specific bacterial taxa at the family and genus levels under different treatments were given as follows.

Effects of consecutive monoculture on rhizosphere bacterial community structure. At the family level, as compared with CK, $P$. heterophylla cultivation (NP and $\mathrm{CM}$ ) led to a significant decrease in the relative abundances of the families Haliangiaceae and Nitrospiraceae but a significant increase in the family Alcaligenaceae (Supplementary Fig. S4). With the extended monoculture, the relative abundances of some other families such as Xanthomonadaceae, Phyllobacteriaceae and Sphingobacteriaceae significantly increased (CK versus $\mathrm{CM}$ ). As compared with NP, the relative abundances of the families Xanthomonadaceae, Chitinophagaceae, and Phyllobacteriaceae significantly increased in CM but the abundances of the families Acidobacteriaceae (subgroup 1), Xanthobacteraceae, and Burkholderiaceae significantly decreased in CM (Fig. 5A). Furthermore, SIMPER analysis based on the relative abundances of the genera showed the top genera that contributed $70 \%$ to the dissimilarity between NP and CM.

Effects of microbial fertilizer application on rhizosphere bacterial community structure. As compared with the $\mathrm{CM}$ treatment, the MF treatment significantly increased the relative abundance of the family Burkholderiaceae while decreasing the relative abundances of the families Xanthomonadaceae, Chitinophagaceae, and Phyllobacteriaceae (Fig. 5B). Furthermore, several genera belonging to the abovementioned families contributed to the observed differences between

Table 1. Changes in soil physicochemical parameters under different treatments ${ }^{\mathrm{z}}$

\begin{tabular}{|c|c|c|c|c|c|c|c|}
\hline Treatments & Total N $\left(\mathbf{g} \cdot \mathrm{kg}^{-1}\right)$ & Available N (mg $\left.\cdot \mathrm{kg}^{-1}\right)$ & Total P $\left(\mathrm{g} \cdot \mathrm{kg}^{-1}\right)$ & Available P $\left(\mathrm{mg} \cdot \mathrm{kg}^{-1}\right)$ & Total K $\left(\mathrm{g} \cdot \mathrm{kg}^{-1}\right)$ & Available K $\left(\mathrm{mg} \cdot \mathrm{kg}^{-1}\right)$ & $\mathbf{p H}$ \\
\hline CK & $2.19 \mathrm{~b}$ & $201.50 \mathrm{e}$ & $0.24 \mathrm{e}$ & $62.10 \mathrm{~d}$ & $8.00 \mathrm{c}$ & $357.97 \mathrm{~b}$ & $5.21 \mathrm{~b}$ \\
\hline NP & $2.16 \mathrm{~b}$ & $229.35 \mathrm{c}$ & $0.33 \mathrm{c}$ & $82.78 b$ & $8.21 \mathrm{~b}$ & $121.47 \mathrm{e}$ & $4.49 \mathrm{~d}$ \\
\hline $\mathrm{CM}$ & $2.50 \mathrm{a}$ & $271.09 \mathrm{~b}$ & $0.37 \mathrm{~b}$ & $81.97 \mathrm{~b}$ & $7.39 \mathrm{e}$ & $203.77 \mathrm{~d}$ & $4.41 \mathrm{e}$ \\
\hline $\mathrm{MF}$ & $2.16 \mathrm{~b}$ & $215.50 \mathrm{~d}$ & $0.30 \mathrm{~d}$ & $72.48 \mathrm{c}$ & $7.81 \mathrm{~d}$ & $236.10 \mathrm{c}$ & $4.52 \mathrm{c}$ \\
\hline PR & $1.98 \mathrm{c}$ & $302.28 \mathrm{a}$ & $0.54 \mathrm{a}$ & $114.03 \mathrm{a}$ & $8.97 \mathrm{a}$ & $400.99 \mathrm{a}$ & $5.30 \mathrm{a}$ \\
\hline
\end{tabular}

z Total N, available N, total $\mathrm{P}$, available $\mathrm{P}$, total $\mathrm{K}$, and available $\mathrm{K}$ represent total nitrogen, available (alkali-hydrolyzable) nitrogen, total phosphorus, available phosphorus, total potassium, and available potassium, respectively. CK, NP, and CM represent the control with no Pseudostellaria heterophylla cultivation, the newly planted plots, and 2-year monocultured plots, respectively. MF represents the 1-year cultivated plots first treated with the microbial fertilizer FAFU and then replanted with $P$. heterophylla and PR represents $P$. heterophylla-rice- $P$. heterophylla rotation plots. Different letters in columns represent significant differences among different treatments (least significant difference test, $P<0.05$ ).
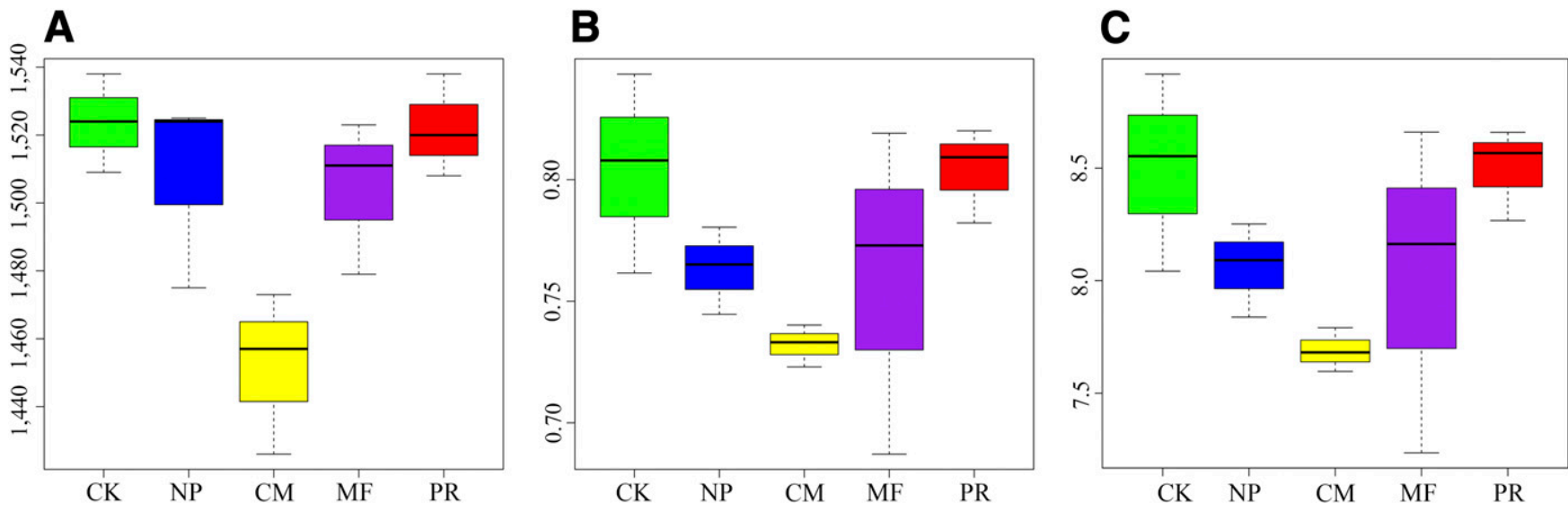

Fig. 3. Calculations of A, operational taxonomic unit number; B, Pielou's evenness; and C, Shannon diversity indices of soil bacterial community in different treatments. CK, NP, and CM represent the control with no Pseudostellaria heterophylla cultivation, the newly planted plots, and 2-year monocultured plots, respectively. MF represents the 1-year cultivated plots first treated with the microbial fertilizer FAFU and then replanted with $P$. heterophylla and PR represents $P$. heterophylla-rice-P. heterophylla rotation plots. 
$\mathrm{MF}$ and $\mathrm{CM}$, and showed a similar trend after microbial fertilizer application. In particular, the relative abundance of Burkholderia spp. (belonging to the family Burkholderiaceae) significantly increased after MF treatment.

Effects of paddy-upland rotation on rhizosphere bacterial community structure. As compared with the CM treatment, the PR treatment significantly increased the relative abundances of the families of Acidobacteriaceae (subgroup 1), Solibacteraceae (subgroup 3), and Nitrosomonadaceae while decreasing the relative abundances of the families Xanthomonadaceae, Chitinophagaceae, Alcaligenaceae, and Phyllobacteriaceae (Fig. 5C). The top genera with $70 \%$ cumulative contribution to the dissimilarity between $\mathrm{CM}$ and $\mathrm{PR}$ are listed in Supplementary Table S1.

Shifts in the predicted functional potentials of rhizosphere bacterial community under different treatments. PICRUSt analysis was carried out to predict the functional potentials of the soil bacterial community's metagenome from the $16 \mathrm{~S}$ rRNA gene information. The results showed the difference in predicted functional potentials of rhizosphere microbial communities between relatively healthy (CK, NP, MF, and PR) and unhealthy (CM) plots (Fig. 6; Supplementary Fig. S5). In total, 23 pathways (at KEGG level 2) were predicted. Among them, 8, 5, and 12 predicted pathways were found to have significant statistical differences $(P<$ 0.05 ) between NP and CM, CM and MF, and CM and PR, respectively (Fig. 6). Several predicted pathways were significantly enriched in CM in comparison with relatively healthy soil (NP, $\mathrm{MF}$, and PR); among them, genes associated with signal transduction, membrane transport, and replication and repair. However, the opposite was true for genes presumptively involved in carbohydrate metabolism (Fig. 6).

\section{Discussion}

Replant disease caused by negative PSF commonly occurs in extensive and monocultural agriculture, and plays a significant role in mediating individual plant performance. In this study, as compared with the NP treatment, the CM treatment led to a significant decline in the yield of $P$. heterophylla tuberous roots, along with an increase in the abundance of $F$. oxysporum. Similarly, the relative abundance of $F$. oxysporum significantly increased under consecutive monoculture of other crops such as peanut, cotton, Rehmannia glutinosa, and Panax notoginseng, which was considered to be the key factor limiting production in the monoculture regime (Dong et al. 2016; $\mathrm{Li}$ et al. 2014b, 2015; Wu et al. 2018a). In recent years, numerous studies have appeared illustrating the mutual relationship between plants and their associated microorganisms (Browne et al. 2018; Lundberg and Teixeira 2018; Sánchez-Cañizares et al. 2017; Wei et al. 2018). Plant-associated microorganisms can be considered to be part of the plant's pan-genome or second genome, which are crucial for plant health and productivity (Berendsen et al. 2012; Tkacz and Poole 2015). Therefore, barcoded pyrosequencing of 16S rDNA amplicons combined with PICRUSt analysis was applied to study the shifts in soil bacterial community composition and functional potentials in the rhizosphere of Pseudostellaria heterophylla under consecutive monoculture and different soil amendments.

The findings in this study were in line with many previous studies on other crops that consecutive monoculture decreased the diversity of soil bacterial community (Liu et al. 2014; Tan et al. 2017; Xiong et al. 2015) while the practices of bio-organic fertilizer application or crop rotation increased soil bacterial diversity in the rhizosphere (Ling et al. 2014; Shen et al. 2015b; Yuan et al. 2014; Zhao et al. 2018; Zhou et al. 2017). Moreover, the changes in the soil pH were consistent with the changes in the Shannon diversity index of soil bacterial communities under different treatments. It has been previously reported that the bacterial diversity was influenced by soil pH (Lauber et al. 2009; Rousk et al. 2010). Rousk et al. (2010) indicated that the relative abundance and diversity of bacteria were positively related to soil $\mathrm{pH}$. In addition, soil bacterial diversity is considered to be a critical factor to maintain soil health, and determine soil suppressiveness to soilborne pathogens (Garbeva et al. 2006; Qiu et al. 2012; Wang et al. 2017). Previous studies of Panax notoginseng demonstrated that bacterial diversity was higher in the rhizosphere of healthy plants than in the rhizosphere of diseased plants (Tan et al. 2017; Z. X. Wu et al. 2015). Therefore, the reduction of soil bacterial diversity might result in the decline in soil suppressiveness under $\mathrm{CM}$ treatment, while improved soil bacterial diversity contributed to the increase in the yield of Pseudostellaria heterophylla tuberous roots after MF and PR treatments.

The results of the present study also indicated that consecutive monoculture and different soil amendments significantly influenced bacterial community structure and composition. Similar to our findings, a previous study found that consecutive monoculture of $R$. $\mathrm{glu}$ tinosa led to a significant increase in the relative abundances of
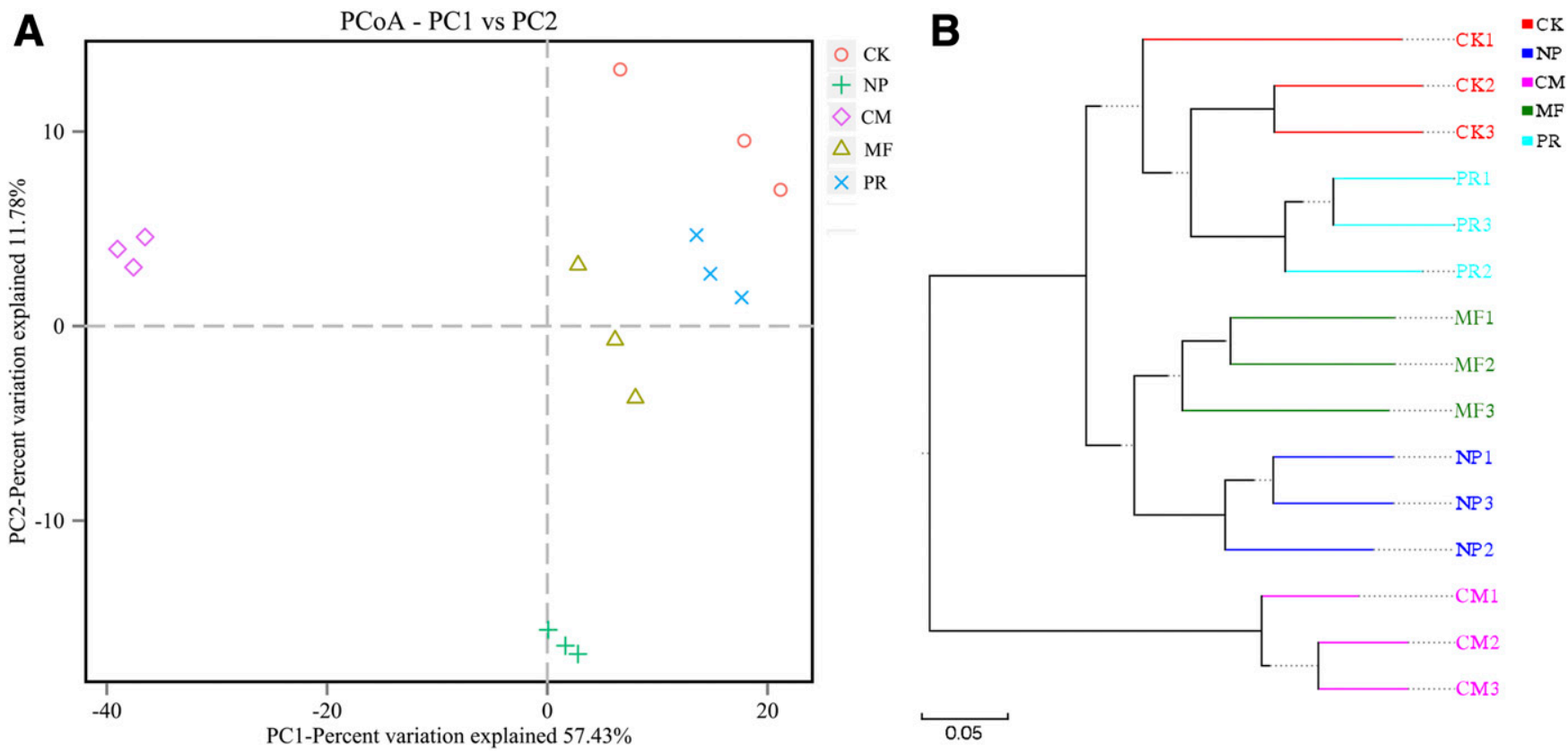

Fig. 4. A, Principal coordinate analysis $(\mathrm{PCOA})$ for principal components $P C 1$ and $P C 2$ and $\mathrm{B}$, unweighted pair-group method with arithmetic means clustering of soil bacterial communities based on the Bray-Curtis distance. CK, NP, and CM represent the control with no Pseudostellaria heterophylla cultivation, the newly planted plots, and 2-year monocultured plots, respectively. MF represents the 1-year cultivated plots first treated with the microbial fertilizer FAFU and then replanted with $P$. heterophylla. Numbers following the treatments in B represent the three replicates. 
Xanthomonadaceae, Sphingomonadaceae, and Alcaligenaceae in the rhizosphere or rhizoplane soil (Wu et al. 2018b), implying that these taxa may be associated with replant disease. Jiang et al. (2017) also demonstrated increased populations of typical pathogenic Xanthomonadaceae bacteria under an apple replanting system. In addition, Sanguin et al. (2009) indicated that the outbreak stage of take-all decline in wheat monoculture was mainly characterized by the prevalence of Proteobacteria, including members of Phyllobacteriaceae and Sphingomonadaceae, which was in line with the findings in this study. However, it should be noted that the families Xanthomonadaceae and Chitinophagaceae were also reported to be associated with soil suppressiveness to wheat head blight (Campos et al. 2016). This discrepancy might be due to the different environmental conditions, host types, pathogen species, and rhizocompartment. Further work is needed to explore the roles of these two families (Xanthomonadaceae and Chitinophagaceae) in rhizosphere of consecutively monocultured

A

NP $\square \mathrm{CM}$

$95 \%$ confidence intervals

Xanthomonadaceae

Acidobacteriaceae (Subgroup 1)

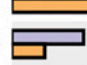

Chitinophagaceae

Xanthobacteraceae $\square$

Microbacteriaceae b

Micrococcaceae 召

Burkholderiaceae $\boldsymbol{p}$

Phyllobacteriaceae b

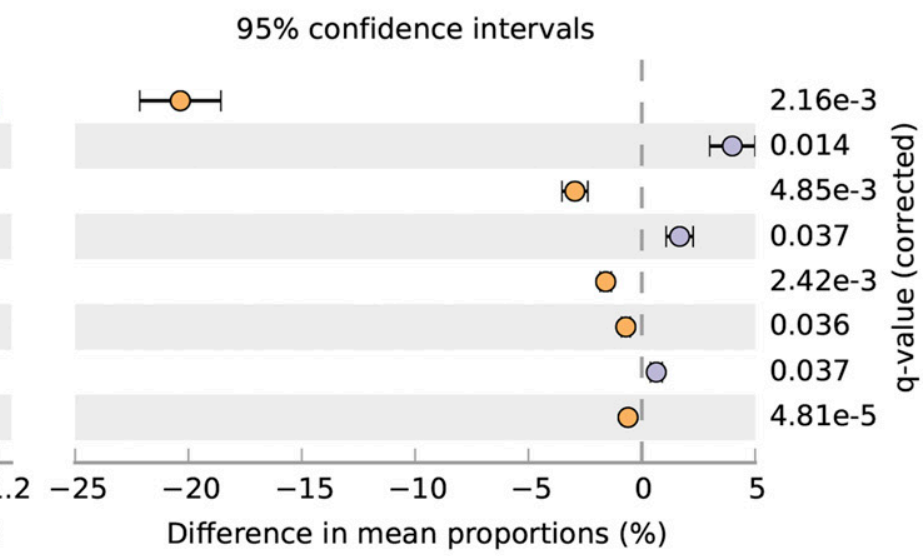

B

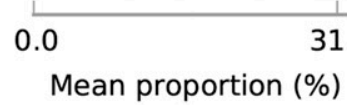

$95 \%$ confidence intervals

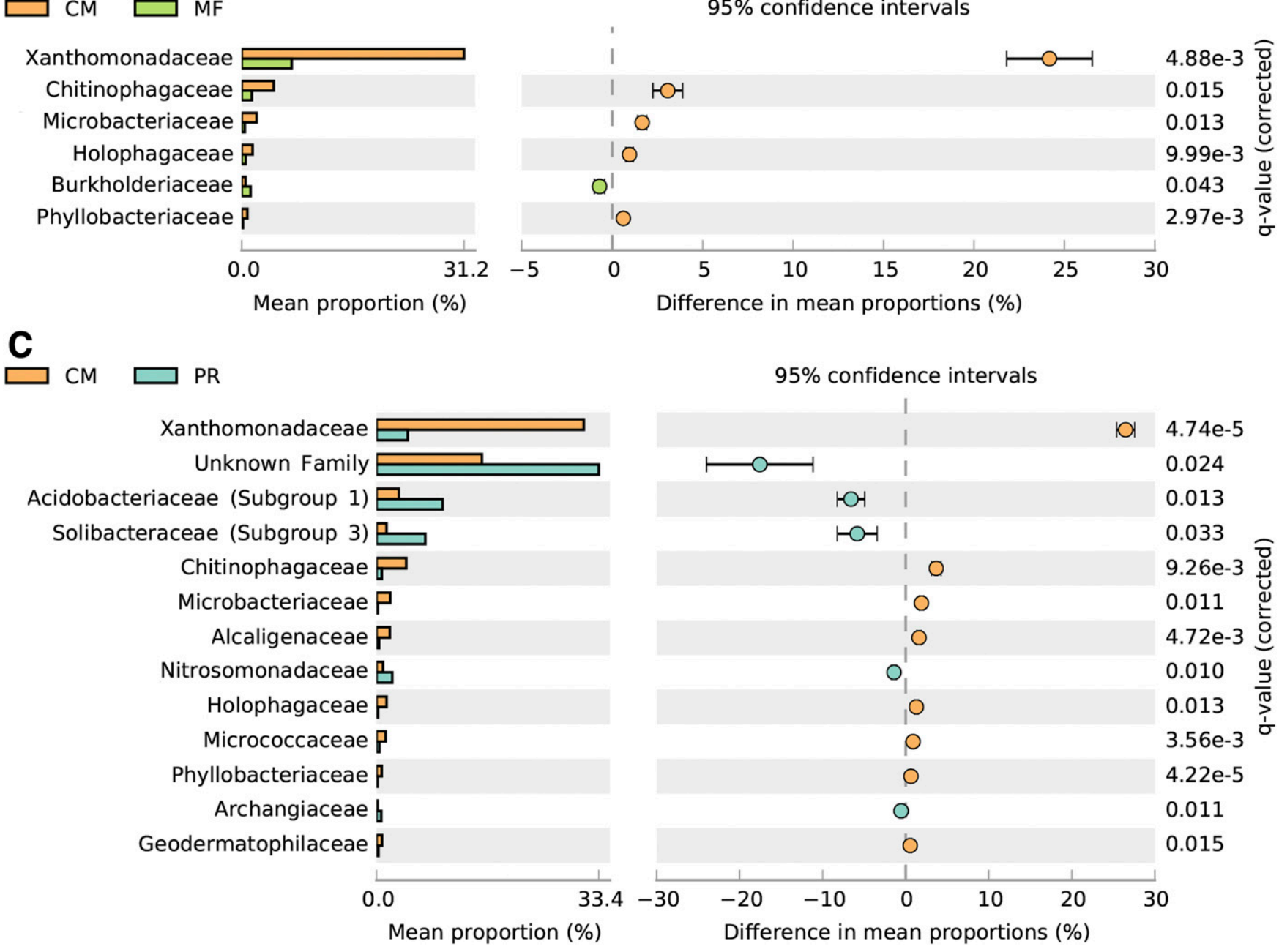

Fig. 5. Mean proportion (\%) and difference in mean proportion (\%) of significantly (Welch's $t$ test, Benjamini-Hochberg false discovery rate correction, $q<0.05$ ) different taxa at the family level between different treatments. NP and CM represent the newly planted and 2-year monocultured plots, respectively; MF represents the 1-year cultivated plots first treated with the microbial fertilizer FAFU and then replanted with Pseudostellaria heterophylla; and PR represents $P$. heterophylla-rice- $P$. heterophylla rotation plots. Statistical Analysis of Metagenomic Profiles extended error bar plots for A, NP versus CM; B, CM versus MF; and C, CM versus PR. The parameters for filtering out were as follows: $q$ value $>0.05$, difference between proportions $<0.5 \%$ or difference between ratios $<2.0$. 
P. heterophylla. By contrast, the relative abundances of families Burkholderiaceae, Xanthobacteraceae, and Acidobacteriaceae significantly decreased under $P$. heterophylla consecutive monoculture. These families were found to be positively correlated with soil suppressiveness on other crops (Campos et al. 2016; Hou et al. 2018; Liu et al. 2016; Sarikhani et al. 2017). In particular, specific members of the family Burkholderiaceae have proved to harbor antagonistic activities against soilborne pathogenic fungi and contribute to soil suppressiveness (Carrión et al. 2018; Kyselková et al. 2009; Mendes et al. 2011). Indeed, certain Burkholderia strains previously isolated from $P$. heterophylla rhizosphere soil showed strong antagonistic activities against $F$. oxysporum, one of the causal agents of root rot disease of

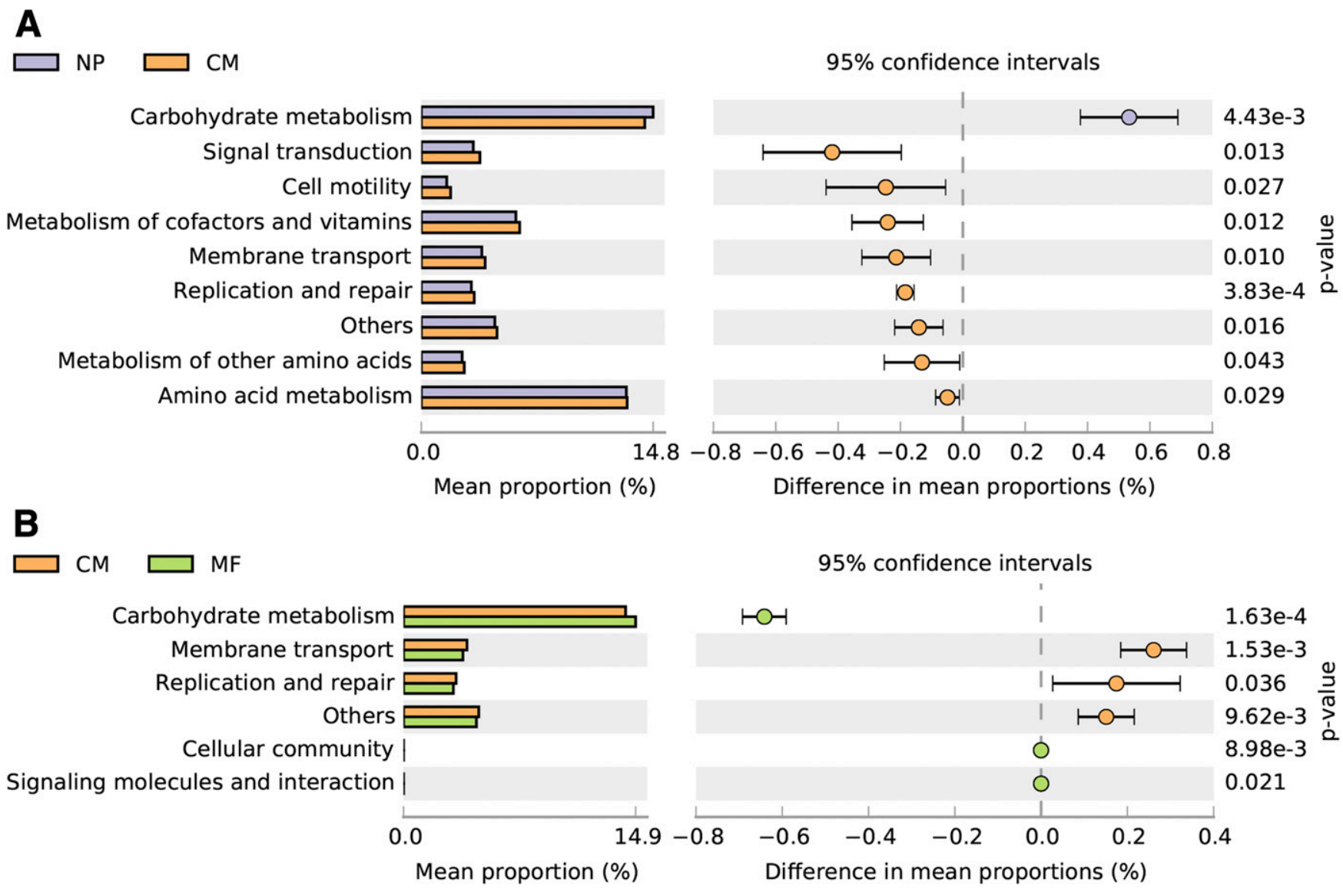

C

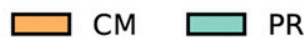

PR

$95 \%$ confidence intervals

Carbohydrate metabolism

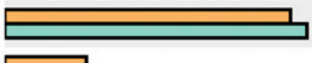

Membrane transport

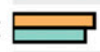

Xenobiotics biodegradation and metabolism

Signal transduction

Energy metabolism

Amino acid metabolism

Metabolism of cofactors and vitamins

Metabolism of other amino acids

Others

Replication and repair

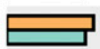

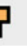

$\rightleftarrows$
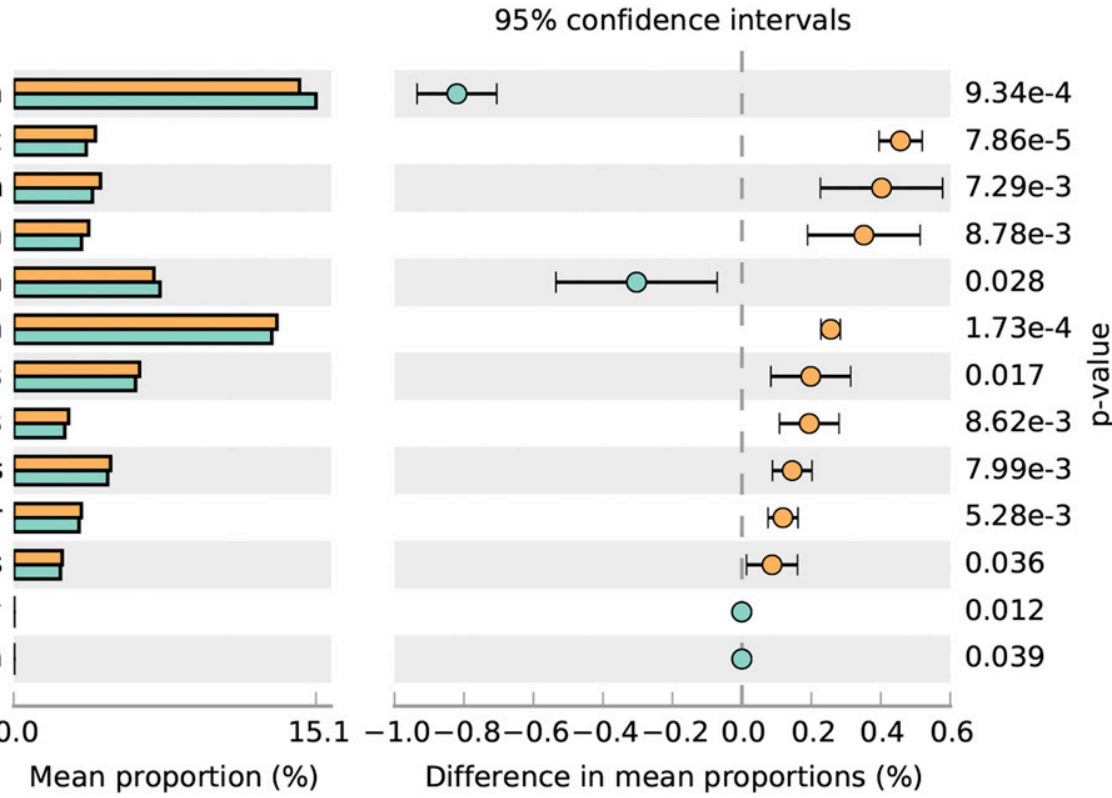

Fig. 6. Mean proportion (\%) and difference in mean proportion (\%) of predicted and significantly (Welch's $t$ test, $P<0.05$ ) different functional categories (at KEGG level 2) between different treatments. The pathways were predicted based on 16S sequences. The predicted metabolic pathways belonging to 'Organismal Systems' and 'Human Diseases' on KEGG database were grouped into 'Others' group. NP and CM represent the newly planted and 2-year monocultured plots, respectively; MF represents the 1-year cultivated plots first treated with the microbial fertilizer FAFU and then replanted with Pseudostellaria heterophylla; and PR represents $P$. heterophylla-rice-P. heterophylla rotation plots. Statistical Analysis of Metagenomic Profiles extended error bar plots for A, NP versus CM; B, CM versus MF; and C, CM versus PR. 
P. heterophylla (L. K. Wu et al. 2016a,b). These results suggested that the decline in the soil suppressiveness due to a reduction in the abundance of beneficial rhizobacteria under consecutive monoculture might be another factor contributing to replant disease of $P$. heterophylla.

Bio-organic fertilizer application has been widely reported to control soilborne disease and improve crop yield by manipulating the soil bacterial community structure (Ling et al. 2014; Wang et al. 2017). In this study, the MF treatment significantly decreased the relative abundances of the CM-enriched families such as Xanthomonadaceae, Chitinophagaceae, and Phyllobacteriaceae while increasing the relative abundance of the family Burkholderiaceae. There was no significant difference between NP and MF in terms of the relative abundance of the family Burkholderiaceae. Wang et al. (2016) found that long-term application of bio-organic fertilizers could significantly increase the relative abundance of Burkholderiales in apple orchard soil. Moreover, the formulated bio-organic fertilizer containing Burkholderia GanoEB2 was proven to effectively suppress Ganoderma disease in oil palm (Ili Nadhrah et al. 2015). Therefore, the increase in $P$. heterophylla yield after the MF treatment might be due to the enrichment of specific antagonistic bacteria (i.e., Burkholderia spp.) in addition to the improvement of soil bacterial diversity.

Increasing plant diversity by intercropping or rotation has also been widely applied as an efficient strategy to reduce soilborne diseases by reshaping the soil microbiome (Latz et al. 2012; Li et al. 2018; Peralta et al. 2018; Zhou et al. 2017). Li et al. (2018) found that intercropping with Atractylodes lancea significantly decreased the relative abundances of the families Xanthomonadaceae, Alcaligenaceae, and Chitinophagaceae in the peanut rhizosphere, which was consistent with the findings under paddy-upland rotation in the current study. However, unlike with the MF treatment, the PR treatment did not increase the relative abundance of the family Burkholderiaceae but mainly increased the relative abundances of the families of Acidobacteriaceae (subgroup 1), Solibacteraceae (subgroup 1), and Archangiaceae, which might be due to the creation of anaerobic conditions when rice was planted after the preceding $P$. heterophylla harvest (Lopes et al. 2014; Maarastawi et al. 2018). Rice cropping was reported to be positively correlated with the abundance of members of the orders Acidobacteriales and Solibacterales (Lopes et al. 2014). Moreover, Hou et al. (2018) indicated that Acidobacteria subgroups GP16 and GP6 played essential roles against pathogens in paddy-upland cropping systems. In addition, it was found that paddy-upland rotation obviously increased more microbial diversity than upland rotation, and improved soil health for sustainable agriculture (Hou et al. 2018). Therefore, the increase in $P$. heterophylla yield after PR treatment might be closely associated with the eminence of paddy-upland rotation in promoting bacterial diversity and modulating specific microbial composition (i.e., Acidobacteriales and Solibacterales) due to the switching between flooding and drying under anaerobic and aerobic conditions.

Furthermore, the PICRUSt approach was applied to predict the functional potentials of the microbial communities in the rhizosphere of $P$. heterophylla and the results revealed the difference in predicted functions between relatively healthy (CK, NP, MF, and PR) and unhealthy (CM) plots. Z. X. Wu et al. (2016) found that relative abundance of gene families related to carbohydrate metabolism tended to be higher in the rhizosphere of healthy $P$. notoginseng, while those related to signal transduction, membrane transport, cell motility, and metabolism of other amino acids tended to be higher in the rhizosphere of diseased plants, which was in line with the findings in this study. In addition, a previous study on $R$. glutinosa rhizosphere soil using shotgun metagenomic sequencing showed that the levels of the clusters of membrane transport (i.e., $\mathrm{ABC}$ transporters) and DNA repair were higher in consecutively monocultured soil than in newly planted soil for harmful or indicator microorganisms (Wu et al. 2018c). Among them, membrane transport was reported to be associated with defense mechanisms, resistance to toxic or

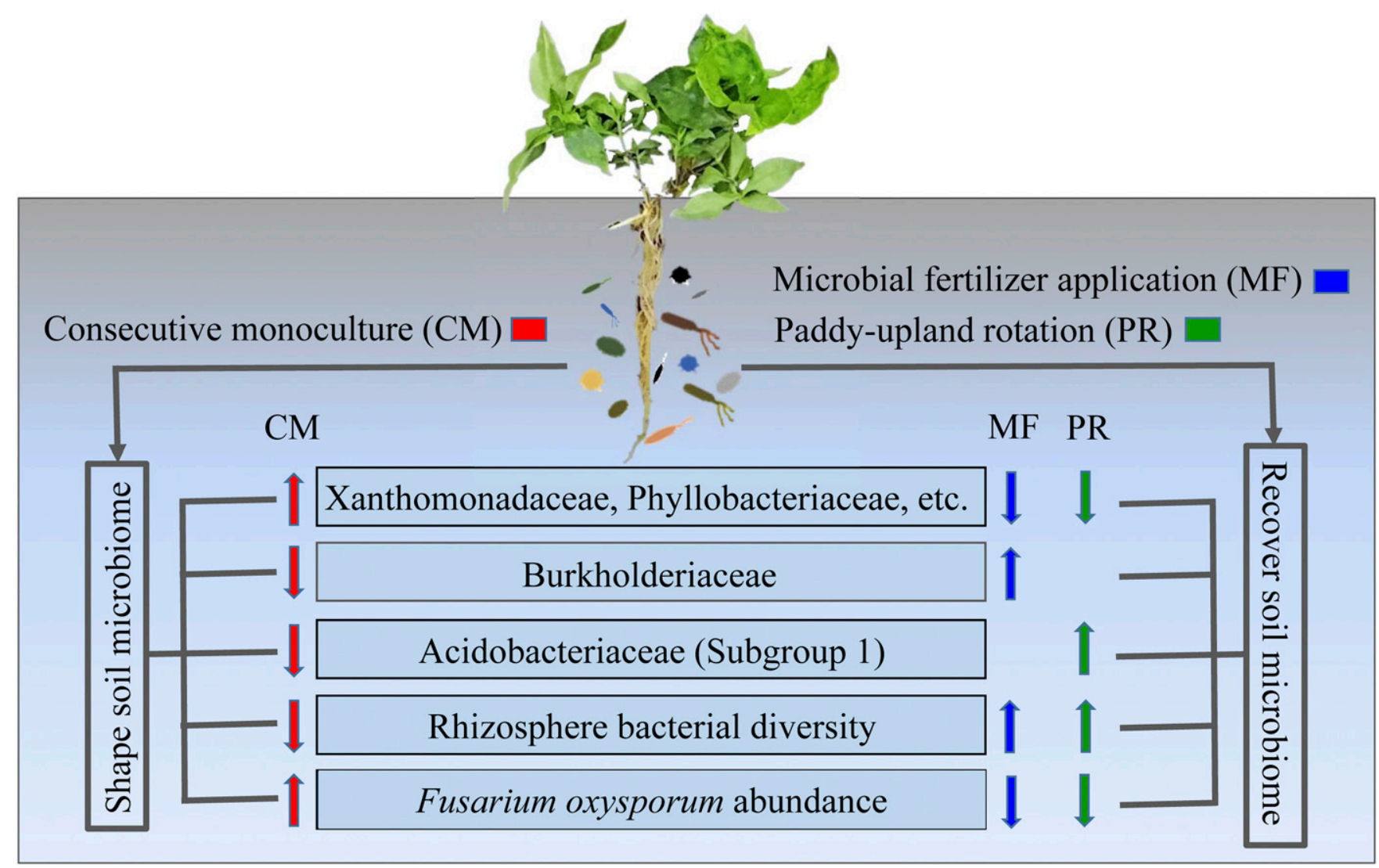

Fig. 7. Schematic representation of the proposed microbial community responses to Pseudostellaria heterophylla consecutive monoculture and different soil amendments. Upward and downward arrows represent the upregulation and downregulation of specific microbial taxa under different treatments, respectively. CM represents the 2-year consecutively monocultured plots (as indicated by red arrows), MF represents the 1-year cultivated plots first treated with the microbial fertilizer FAFU and then replanted with $P$. heterophylla (as indicated by blue arrows), and PR represents $P$. heterophylla-rice-P. heterophylla rotation plots (as indicated by green arrows). 
antimicrobial compounds, and bacterial rapid growth by regulating the transport function across cell membranes (Wu et al. 2018c; $\mathrm{Z}$. $X$. Wu et al. 2016). Bacterial motility is involved in root colonization by mediating the movement toward the root in soil and spread in the root after successful infection (Z. X. Wu et al. 2016). Therefore, the higher abundances of membrane transport, cell motility, and DNA repair in $\mathrm{CM}$ might contribute to the root colonization and survival for certain bacterial pathogens in the monocultured soil of $P$. heterophylla. It should be noted, however, that the PICRUSt approach is limited by the number of available genomes, although PICRUSt analysis has been shown to replicate metagenomes to a high degree of accuracy, and gene expression of soil microbial community cannot be captured by this approach as well (Jiang et al. 2016; Langille et al. 2013; Suenaga 2012).

In conclusion, the findings in this study revealed that the replant disease of $P$. heterophylla was closely associated with the dysbiosis of rhizosphere microbiota. The MF and PR treatments could increase soil bacterial diversity and reshape the rhizosphere bacterial community to a similar status with NP or CK (Fig. 7). Both practices can be chosen to alleviate $P$. heterophylla replant disease, depending on the water availability in the geo-authentic production areas. We also showed that the MF and PR treatments recovered the soil microbiome through different ways; for example, the introduction of beneficial microorganisms (in MF) or the switching between flooded soil and upland soil conditions (in PR). Our results reinforce the importance of the rhizosphere microbiome in maintaining soil quality and plant health. Further work is needed to investigate functional gene expression of the rhizosphere microbiome with soil metatranscriptomics or metaproteomics and to link the functional genes of uncultured organisms to phylogenetic groups.

\section{Literature Cited}

Berendsen, R. L., Pieterse, C. M., and Bakker, P. A. 2012. The rhizosphere microbiome and plant health. Trends Plant Sci. 17:478-486.

Bolger, A. M., Lohse, M., and Usadel, B. 2014. Trimmomatic: A flexible trimmer for Illumina sequence data. Bioinformatics 30:2114-2120.

Breidenbach, B., Brenzinger, K., Brandt, F. B., Blaser, M. B., and Conrad, R. 2017. The effect of crop rotation between wetland rice and upland maize on the microbial communities associated with roots. Plant Soil 419:435-445.

Browne, G., Ott, N., Poret-Peterson, A., Gouran, H., and Lampinen, B. 2018. Efficacy of anaerobic soil disinfestation for control of Prunus replant disease. Plant Dis. 102:209-219.

Campos, S. B., Lisboa, B. B., Camargo, F. A., Bayer, C., Sczyrba, A., Dirksen, P., Albersmeier, A., Kalinowski, J., Beneduzi, A., Costa, P. B., Passaglia, L. M. P., Vargas, L. K., and Wendisch, V. F. 2016. Soil suppressiveness and its relations with the microbial community in a Brazilian subtropical agroecosystem under different management systems. Soil Biol. Biochem. 96:191-197.

Caporaso, J. G., Kuczynski, J., Stombaugh, J., Bittinger, K., Bushman, F. D., Costello, E. K., Fierer, N., Peña, A. G., Goodrich, J. K., Gordon, J. I., Huttley, G. A., Kelley, S. T., Knights, D., Koenig, J. E., Ley, R. E., Lozupone, C. A., McDonald, D., Muegge, B. D., Pirrung, M., Reeder, J., Sevinsky, J. R., Turnbaugh, P. J., Walters, W. A., Widmann, J., Yatsunenko, T., Zaneveld, J., and Knight, R. 2010. QIIME allows analysis of highthroughput community sequencing data. Nat. Methods 7:335-336.

Carrión, V. J., Cordovez, V., Tyc, O., Etalo, D. W., de Bruijn, I., de Jager, V. C. L., Medema, M. H., Eberl, L., and Raaijmakers, J. M. 2018. Involvement of Burkholderiaceae and sulfurous volatiles in disease-suppressive soils. ISME J. 12:2307-2321.

Chen, J., Wu, L. K., Xiao, Z. X., Wu, Y. H., Wu, H. M., Qin, X. J., Wang, J. Y., Wei, X. Y., Khan, M. U., Lin, S., and Lin, W. X. 2017. Assessment of the diversity of Pseudomonas spp. and Fusarium spp. in Radix pseudostellariae rhizosphere under monoculture by combining DGGE and quantitative PCR. Front. Microbiol. 8:1748.

Chen, T., Lin, S., Wu, L. K., Lin, W. X., and Sampietro, D. A. 2015. Soil sickness: Current status and future perspectives. Allelopathy J. 36:167-196.

Dong, L. L., Xu, J., Feng, G. Q., Li, X. W., and Chen, S. L. 2016. Soil bacterial and fungal community dynamics in relation to Panax notoginseng death rate in a continuous cropping system. Sci. Rep. 6: Article 31802

Edgar, R. C., Haas, B. J., Clemente, J. C., Quince, C., and Knight, R. 2011. UCHIME improves sensitivity and speed of chimera detection. Bioinformatics 27:2194-2200

Garbeva, P., Postma, J., Van Veen, J. A., and Van Elsas, J. D. 2006. Effect of aboveground plant species on soil microbial community structure and its impact on suppression of Rhizoctonia solani AG3. Environ. Microbiol. 8:233-246.

Gaydon, D. S., Probert, M. E., Buresh, R. J., Meinke, H., Suriadi, A., Dobermann, A., Bouman, B., and Timsina, J. 2012. Rice in cropping systems-Modelling transitions between flooded and non-flooded soil environments. Eur. J. Agron. 39:9-24.

Gómez Expósito, R., de Bruijn, I., Postma, J., and Raaijmakers, J. M. 2017. Current insights into the role of rhizosphere bacteria in disease suppressive soils. Front. Microbiol. 8:2529.

Hou, P. F., Chien, C. H., Chiang-Hsieh, Y. F., Tseng, K. C., Chow, C. N., Huang, H. J., and Chang, W. C. 2018. Paddy-upland rotation for sustainable agriculture with regards to diverse soil microbial community. Sci. Rep. 8: Article 7966.

Hu, L. F., Robert, C. A., Cadot, S., Zhang, X., Ye, M., Li, B. B., Manzo, D. Chervet, N., Steinger, T., van der Heijden, M. G. A., Schlaeppi, K., and Erb, M. 2018. Root exudate metabolites drive plant-soil feedbacks on growth and defense by shaping the rhizosphere microbiota. Nat. Commun. 9: Article 2738.

Hua, Y. J., Wang, S. N., Liu, Z. X., Liu, X. H., Zou, L. S., Gu, W., Luo, Y. Y., and Liu, J. X. 2016. Transcriptomic analysis of Pseudostellariae Radix from different fields using RNA-seq. Gene 588:7-18.

Huang, L. F., Song, L. X., Xia, X. J., Mao, W. H., Shi, K., Zhou, Y. H., and Yu, J. Q. 2013. Plant-soil feedbacks and soil sickness: From mechanisms to application in agriculture. J. Chem. Ecol. 39:232-242.

Ili Nadhrah, N., Nulit, R., Nurrashyeda, R., and Idris, A. S. 2015. Effect of formulated bioorganic containing Burkholderia Ganoeb2 in suppressing Ganoderma disease in oil palm seedlings. Plant Prot. Sci. 51:80-87.

Jiang, J. H., Song, Z., Yang, X. T., Mao, Z. Q., Nie, X. H., Guo, H., and Peng, X. W. 2017. Microbial community analysis of apple rhizosphere around Bohai Gulf. Sci. Rep. 7: Article 8918.

Jiang, L. F., Song, M., Yang, L., Zhang, D. Y., Sun, Y. T., Shen, Z. G., Luo, C. L. and Zhang, G. 2016. Exploring the influence of environmental factors on bacterial communities within the rhizosphere of the $\mathrm{Cu}$-tolerant plant, Elsholtzia splendens. Sci. Rep. 6: Article 36302.

Kaisermann, A., Vries, F. T., Griffiths, R. I., and Bardgett, R. D. 2017. Legacy effects of drought on plant-soil feedbacks and plant-plant interactions. New Phytol. 215:1413-1424

Kyselková, M., Kopecký, J., Frapolli, M., Défago, G., Ságová-Marečková, M. Grundmann, G. L., and Moënne-Loccoz, Y. 2009. Comparison of rhizobacterial community composition in soil suppressive or conducive to tobacco black root rot disease. ISME J. 3:1127-1138.

Langille, M. G., Zaneveld, J., Caporaso, J. G., McDonald, D., Knights, D., Reyes, J. A. Clemente, J. C., Burkepile, D. E., Vega Thurber, R. L., Knight, R., Beiko, R. G., and Huttenhower, C. 2013. Predictive functional profiling of microbial communities using 16S rRNA marker gene sequences. Nat. Biotechnol. 31:814-821.

Lareen, A., Burton, F., and Schäfer, P. 2016. Plant root-microbe communication in shaping root microbiomes. Plant Mol. Biol. 90:575-587.

Latz, E., Eisenhauer, N., Rall, B. C., Allan, E., Roscher, C., Scheu, S., and Jousset, A. 2012. Plant diversity improves protection against soil-borne pathogens by fostering antagonistic bacterial communities. J. Ecol. 100:597-604.

Lauber, C. L., Hamady, M., Knight, R., and Fierer, N. 2009. Pyrosequencingbased assessment of soil $\mathrm{pH}$ as a predictor of soil bacterial community structure at the continental scale. Appl. Environ. Microbiol. 75:5111-5120.

Li, X. G., de Boer, W., Zhang, Y. N., Ding, C. F., Zhang, T. L., and Wang, X. X 2018. Suppression of soil-borne Fusarium pathogens of peanut by intercropping with the medicinal herb Atractylodes lancea. Soil Biol. Biochem. 116:120-130.

Li, X. G., Ding, C. F., Hua, K., Zhang, T. L., Zhang, Y. N., Zhao, L., Zhang, Y. N., Zhao, L., Yang, Y. R., Liu, J. G., and Wang, X. X. 2014a. Soil sickness of peanuts is attributable to modifications in soil microbes induced by peanut root exudates rather than to direct allelopathy. Soil Biol. Biochem. 78:149-159.

Li, X. G., Ding, C. F., Zhang, T. L., and Wang, X. X. 2014b. Fungal pathogen accumulation at the expense of plant-beneficial fungi as a consequence of consecutive peanut monoculturing. Soil Biol. Biochem. 72:11-18

Li, X. G., Zhang, Y. N., Ding, C. F., Jia, Z. J., He, Z. L., Zhang, T. L., and Wang, X. X. 2015. Declined soil suppressiveness to Fusarium oxysporum by rhizosphere microflora of cotton in soil sickness. Biol. Fertil. Soils 51:935-946.

Lievens, B., Brouwer, M., Vanachter, A. C., Lévesque, C. A., Cammue, B. P., and Thomma, B. P. 2005. Quantitative assessment of phytopathogenic fungi in various substrates using a DNA macroarray. Environ. Microbiol. 7:1698-1710.

Ling, N., Deng, K. Y., Song, Y., Wu, Y. C., Zhao, J., Raza, W., Huang, Q. W., and Shen, Q. R. 2014. Variation of rhizosphere bacterial community in watermelon continuous mono-cropping soil by long-term application of a novel bioorganic fertilizer. Microbiol. Res. 169:570-578.

Liu, X., Zhang, J., Gu, T., Zhang, W., Shen, Q., Yin, S., and Qiu, H. 2014 Microbial community diversities and taxa abundances in soils along a sevenyear gradient of potato monoculture using high throughput pyrosequencing approach. PLoS One 9:e86610.

Liu, X. J., Zhang, S., Jiang, Q., Bai, Y., Shen, G., Li, S., and Ding, W. 2016. Using community analysis to explore bacterial indicators for disease suppression of tobacco bacterial wilt. Sci. Rep. 6: Article 36773

Lopes, A. R., Manaia, C. M., and Nunes, O. C. 2014. Bacterial community variations in an alfalfa-rice rotation system revealed by $16 \mathrm{~S}$ rRNA gene 454 pyrosequencing. FEMS Microbiol. Ecol. 87:650-663.

Lundberg, D. S., and Teixeira, P. J. 2018. Root-exuded coumarin shapes the root microbiome. Proc. Natl. Acad. Sci. U.S.A. 115:5629-5631.

Maarastawi, S., Frindte, K., Linnartz, M., and Knief, C. 2018. Crop rotation and straw application impact microbial communities in Italian and Philippine soils and the rhizosphere of Zea mays. Front. Microbiol. 9:1295. 
Magoč, T., and Salzberg, S. L. 2011. FLASH: Fast length adjustment of short reads to improve genome assemblies. Bioinformatics 27:2957-2963.

Mazzola, M., and Manici, L. M. 2012. Apple replant disease: Role of microbial ecology in cause and control. Annu. Rev. Phytopathol. 50:45-65.

Mendes, R., Kruijt, M., De Bruijn, I., Dekkers, E., van der Voort, M., Schneider, J. H., Piceno, Y. M., DeSantis, T. Z., Andersen, G. L., Bakker, P. A., and Raaijmakers, J. M. 2011. Deciphering the rhizosphere microbiome for disease-suppressive bacteria. Science 332:1097-1100.

Pacchioni, R. G., Carvalho, F. M., Thompson, C. E., Faustino, A. L., Nicolini, F., Pereira, T. S., Silva, R. C., Cantão, M. E., Gerber, A., Vasconcelos, A. T., and Agnez-Lima, L. F. 2014. Taxonomic and functional profiles of soil samples from Atlantic forest and Caatinga biomes in northeastern Brazil. MicrobiologyOpen 3:299-315.

Parks, D. H., Tyson, G. W., Hugenholtz, P., and Beiko, R. G. 2014. STAMP: Statistical analysis of taxonomic and functional profiles. Bioinformatics 30: 3123-3124.

Peralta, A. L., Sun, Y., McDaniel, M. D., and Lennon, J. T. 2018. Crop rotational diversity increases disease suppressive capacity of soil microbiomes. Ecosphere 9:e02235.

Poole, P. 2017. Shining a light on the dark world of plant root-microbe interactions. Proc. Natl. Acad. Sci. U.S.A. 114:4281-4283.

Qiu, M. H., Zhang, R. F., Xue, C., Zhang, S. S., Li, S. Q., Zhang, N., and Shen, Q. R. 2012. Application of bio-organic fertilizer can control Fusarium wilt of cucumber plants by regulating microbial community of rhizosphere soil. Biol. Fertil. Soils 48:807-816.

Quast, C., Pruesse, E., Yilmaz, P., Gerken, J., Schweer, T., Yarza, P., Peplies, J., and Glöckner, F. O. 2012. The SILVA ribosomal RNA gene database project: Improved data processing and web-based tools. Nucleic Acids Res. 41: D590-D596.

Rousk, J., Bååth, E., Brookes, P. C., Lauber, C. L., Lozupone, C., Caporaso, J. G., Knight, R., and Fierer, N. 2010. Soil bacterial and fungal communities across a $\mathrm{pH}$ gradient in an arable soil. ISME J. 4:1340-1351.

Sánchez-Cañizares, C., Jorrín, B., Poole, P. S., and Tkacz, A. 2017. Understanding the holobiont: The interdependence of plants and their microbiome. Curr. Opin. Microbiol. 38:188-196.

Sanguin, H., Sarniguet, A., Gazengel, K., Moënne-Loccoz, Y., and Grundmann, G. L. 2009. Rhizosphere bacterial communities associated with disease suppressiveness stages of take-all decline in wheat monoculture. New Phytol. 184:694-707.

Sarikhani, E., Sagova-Mareckova, M., Omelka, M., and Kopecky, J. 2017. The effect of peat and iron supplements on the severity of potato common scab and bacterial community in tuberosphere soil. FEMS Microbiol. Ecol. 93:fiw206.

Schloter, M., Dilly, O., and Munch, J. C. 2003. Indicators for evaluating soil quality. Agric. Ecosyst. Environ. 98:255-262.

Shen, Z. Z., Ruan, Y. Z., Xue, C., Zhong, S. T., Li, R., and Shen, Q. R. 2015a. Soils naturally suppressive to banana Fusarium wilt disease harbor unique bacterial communities. Plant Soil 393:21-33.

Shen, Z. Z., Wang, B. B., Lv, N. N., Sun, Y. F., Jiang, X. Y., Li, R., Ruan, Y. Z., and Shen, Q. R. 2015b. Effect of the combination of bio-organic fertiliser with Bacillus amyloliquefaciens NJN-6 on the control of banana Fusarium wilt disease, crop production and banana rhizosphere culturable microflora. Biocontrol Sci. Technol. 25:716-731.

Suenaga, H. 2012. Targeted metagenomics: A high-resolution metagenomics approach for specific gene clusters in complex microbial communities. Environ. Microbiol. 14:13-22.

Tan, Y., Cui, Y. S., Li, H. Y., Kuang, A. X., Li, X. R., Wei, Y. L., and Ji, X. L. 2017. Diversity and composition of rhizospheric soil and root endogenous bacteria in Panax notoginseng during continuous cropping practices. J. Basic Microbiol. 57:337-344.

Tian, Y., Wang, Q., Zhang, W., and Gao, L. 2016. Reducing environmental risk of excessively fertilized soils and improving cucumber growth by Caragana microphylla-straw compost application in long-term continuous cropping systems. Sci. Total Environ. 544:251-261.

Tkacz, A., and Poole, P. 2015. Role of root microbiota in plant productivity. J. Exp. Bot. 66:2167-2175.

van der Putten, W. H., Bardgett, R. D., Bever, J. D., Bezemer, T. M., Casper, B. B., Fukami, T., Kardol, P., Klironomos, J. N., Kulmatiski, A., Schweitzer, J. A., Suding, K. N., Van de Voorde, T. F. J., and Wardle, D. A. 2013. Plant-soil feedbacks: The past, the present and future challenges. J. Ecol. 101:265-276.

Wang, L., Li, J., Yang, F., E, Y., Raza, W., Huang, Q., and Shen, Q. 2017. Application of bioorganic fertilizer significantly increased apple yields and shaped bacterial community structure in orchard soil. Microb. Ecol. 73:404-416.

Wang, L., Yang, F., E, Y., Yuan, J., Raza, W., Huang, Q., and Shen, Q. 2016. Long-term application of bioorganic fertilizers improved soil biochemical properties and microbial communities of an apple orchard soil. Front. Microbiol. 7:1893.

Wang, Q., Garrity, G. M., Tiedje, J. M., and Cole, J. R. 2007. Naive Bayesian classifier for rapid assignment of rRNA sequences into the new bacterial taxonomy. Appl. Environ. Microbiol. 73:5261-5267.

Wei, W., Yang, M., Liu, Y. X., Huang, H. C., Ye, C., Zheng, J. F., Guo, C. W., Hao, M. W., He, X. H., and Zhu, S. S. 2018. Fertilizer N application rate impacts plant-soil feedback in a sanqi production system. Sci. Total Environ. 633:796-807.
Wu, H. M., Wu, L. K., Zhu, Q., Wang, J. Y., Qin, X. J., Xu, J. H., Kong, L. F., Chen, J., Lin, S., Khan, M. U., Amjad, H., and Lin, W. X. 2017. The role of organic acids on microbial deterioration in the Radix pseudostellariae rhizosphere under continuous monoculture regimes. Sci. Rep. 7: Article 3497.

Wu, L. K., Chen, J., Khan, M. U., Wang, J. Y., Wu, H. M., Xiao, Z. G., Zhang, Z. Y., and Lin, W. X. 2018a. Rhizosphere fungal community dynamics associated with Rehmannia glutinosa replant disease in a consecutive monoculture regime. Phytopathology 108:1493-1500.

Wu, L. K., Chen, J., Wu, H. M., Qin, X. J., Wang, J. Y., Wu, Y. H., Khan, M. U. Lin, S., Xiao, Z. G., Luo, X. M., Zhang, Z. Y., and Lin, W. X. 2016a. Insights into the regulation of rhizosphere bacterial communities by application of bioorganic fertilizer in Pseudostellaria heterophylla monoculture regime. Front. Microbiol. 7:1788.

Wu, L. K., Chen, J., Wu, H. M., Wang, J. Y., Wu, Y. H., Lin, S., Xiao, Z. G., Luo, X. M., Zhang, Z. Y., and Lin, W. X. 2016b. Effects of consecutive monoculture of Pseudostellaria heterophylla on soil fungal community as determined by pyrosequencing. Sci. Rep. 6: Article 26601.

Wu, L. K., Chen, J., Xiao, Z. G., Zhu, X. C., Wang, J. Y., Wu, H. M., Wu, Y. H., Zhang, Z. Y., and Lin, W. X. 2018b. Barcoded pyrosequencing reveals a shift in the bacterial community in the rhizosphere and rhizoplane of Rehmannia glutinosa under consecutive monoculture. Int. J. Mol. Sci. 19:850.

Wu, L. K., Wang, J. Y., Huang, W. M., Wu, H. M., Chen, J., Yang, Y. Q., Zhang, Z. Y., and Lin, W. X. 2015. Plant-microbe rhizosphere interactions mediated by Rehmannia glutinosa root exudates under consecutive monoculture. Sci. Rep. 5: Article 15871

Wu, L. K., Wang, J. Y., Wu, H. M., Chen, J., Xiao, Z. G., Qin, X. J., Zhang, Z. Y., and Lin, W. X. 2018c. Comparative metagenomic analysis of rhizosphere microbial community composition and functional potentials under Rehmannia glutinosa consecutive monoculture. Int. J. Mol. Sci. 19:2394.

Wu, Z. X., Hao, Z. P., Sun, Y. Q., Guo, L. P., Huang, L. Q., Zeng, Y., Wang, Y., Yang, L., and Chen, B. D. 2016. Comparison on the structure and function of the rhizosphere microbial community between healthy and root-rot Panax notoginseng. Appl. Soil Ecol. 107:99-107.

Wu, Z. X., Hao, Z. P., Zeng, Y., Guo, L. P., Huang, L. Q., and Chen, B. D. 2015 Molecular characterization of microbial communities in the rhizosphere soils and roots of diseased and healthy Panax notoginseng. Antonie Leeuwenhoek 108:1059-1074

Xiong, W., Li, Z. G., Liu, H. J., Xue, C., Zhang, R. F., Wu, H. S., Li, R., and Shen, Q. R. 2015. The effect of long-term continuous cropping of black pepper on soil bacterial communities as determined by 454 pyrosequencing. PLoS One 10: e0136946.

$\mathrm{Xu}, \mathrm{H}$. L. 2001. Effects of a microbial inoculant and organic fertilizers on the growth, photosynthesis and yield of sweet corn. J. Crop Prod. 3 183-214

Yang, Y. R., Li, X. G., Liu, J. G., Zhou, Z. G., Zhang, T. L., and Wang, X. X. 2017. Bacterial diversity as affected by application of manure in red soils of subtropical China. Biol. Fertil. Soils 53:639-649.

Yao, H. Y., Jiao, X. D., and Wu, F. Z. 2006. Effects of continuous cucumber cropping and alternative rotations under protected cultivation on soil microbial community diversity. Plant Soil 284:195-203.

Yuan, S. F., Wang, L. L., Wu, K., Shi, J. X., Wang, M. S., Yang, X. M., Shen, Q. R., and Shen, B. 2014. Evaluation of Bacillus-fortified organic fertilizer for controlling tobacco bacterial wilt in greenhouse and field experiments. Appl. Soil Ecol. 75:86-94.

Zhang, Z. Y., Lin, W. X., Yang, Y. H., Chen, H., and Chen, X. J. 2011. Effects of consecutively monocultured Rehmannia glutinosa L. on diversity of fungal community in rhizospheric soil. Agric. Sci. China 10:1374-1384.

Zhao, J., Li, Y. L., Wang, B. Y., Huang, X. Q., Yang, L., Lan, T., Zhang, J. B., and Cai, Z. C. 2017. Comparative soil microbial communities and activities in adjacent Sanqi ginseng monoculture and maize-Sanqi ginseng systems. Appl. Soil Ecol. 120:89-96.

Zhao, J., Liu, J., Liang, H., Huang, J., Chen, Z., Nie, Y. J., Wang, C. B., and Wang, Y. G. 2018. Manipulation of the rhizosphere microbial community through application of a new bio-organic fertilizer improves watermelon quality and health. PLoS One 13:e0192967.

Zhao, J., Zhang, R. F., Xue, C., Xun, W. B., Sun, L., Xu, Y. C., and Shen, Q. R. 2014. Pyrosequencing reveals contrasting soil bacterial diversity and community structure of two main winter wheat cropping systems in China. Microb. Ecol. 67:443-453.

Zhao, Y. P., Wu, L. K., Chu, L. X., Yang, Y. Q., Li, Z. F., Azeem, S., Zhang, Z. X. Fang, C. X., and Lin, W. X. 2015. Interaction of Pseudostellaria heterophylla with Fusarium oxysporum f. sp. heterophylla mediated by its root exudates in a consecutive monoculture system. Sci. Rep. 5: Article 8197.

Zhou, W., Lv, T.-F., Chen, Y., Westby, A. P., and Ren, W.-J. 2014. Soil physicochemical and biological properties of paddy-upland rotation: A review. Sci. World J. 2014: Article 856352.

Zhou, X. G., Liu, J., and Wu, F. Z. 2017. Soil microbial communities in cucumber monoculture and rotation systems and their feedback effects on cucumber seedling growth. Plant Soil 415:507-520.

Zhou, X. G., and Wu, F. Z. 2018. Vanillic acid changed cucumber (Cucumis sativus L.) seedling rhizosphere total bacterial, Pseudomonas and Bacillus spp. communities. Sci. Rep. 8: Article 4929. 